\title{
Influence of pre-existing dementia on the risk of post-stroke epileptic seizures
}

\author{
C Cordonnier, H Hénon, P Derambure, F Pasquier, D Leys
}

J Neurol Neurosurg Psychiatry 2005;76:1649-1653. doi: 10.1136/jnnp.2005.064535

See end of article for authors' affiliations .....................

Correspondence to: Professor D Leys, Department of Neurology, Stroke Unit, Hôpital Roger Salengro, F-59037 Lille,

France; dleys@chru-lille.fr

Received 31 January 2005 Revised version received 9 May 2005

Accepted 16 May 2005
Background: Seizures occur in 10\% of stroke patients, but their predictors have not been clearly identified. Pre-existing dementia is present in 12-16\% of stroke patients and, at the community level, patients with dementia have increased risk of seizures. However, the question of whether pre-existing dementia is associated with a higher risk of seizures after stroke has never been studied.

Aim: To evaluate whether pre-existing dementia is associated with an increased risk of seizures after stroke. Methods: The study was conducted on 202 consecutive stroke patients recruited to the Lille stroke/ dementia study (97 men; median age, 75 years; range, 42-100). Pre-stroke cognitive functions were evaluated using the Informant Questionnaire on Cognitive Decline in the Elderly, with a cutoff value of 104 for the diagnosis of dementia. Seizures were defined as early seizures when occurring within seven days of stroke onset, and as late seizures when occurring more than seven days after stroke.

Results: Of 202 patients, 33 (16.3\%) met the criteria for pre-existing dementia, and 11 (5.4\%) developed early seizures. During 289 person-years of follow up, 14 patients developed late seizures, resulting in an incidence rate of 4.8 new cases/100 person-years. Pre-existing dementia was not associated with the occurrence of early seizures, but was independently associated with the occurrence of late seizures (adjusted odds ratio, 4.66 ; 95\% confidence interval, 1.34 to 16.21 ).

Conclusion: Stroke patients with pre-existing dementia have an increased risk of late seizures. Any factor increasing the risk of seizures (drugs, metabolic changes) should be avoided in these patients.
E pileptic seizures occur within two weeks of stroke onset in $1.8-8 \%$ of patients, ${ }^{1-7}$ and late onset seizures occur in $3.2-4.5 \%$ of patients. ${ }^{3} 7$ Risk factors for seizures in stroke patients are not clearly identified, ${ }^{8}$ but a cortical lesion ${ }^{679-11}$ and a more severe stroke ${ }^{4}$ are associated with a higher risk.

In white populations, $12-16 \%$ of stroke patients have preexisting dementia. ${ }^{12-14}$ Hospital based ${ }^{15}$ and population based studies $^{16}$ have shown that patients with dementia have an increased risk of seizures compared with the general population. However, the question of whether stroke patients with pre-existing dementia are at increased risk for poststroke seizures has never been investigated.

The aim of our study was to evaluate the influence of preexisting dementia on the risk of seizures after stroke.

\section{METHOD}

The Lille stroke/dementia cohort was recruited over a 28 week period between November 1995 and January 1996 for a prospective systematic evaluation of dementia in patients with stroke. ${ }^{12}{ }^{17}$ All consecutive patients with stroke admitted to the acute stroke unit of the Lille University Hospital, France were eligible, except those with transient ischaemic attack (TIA), cerebral venous thrombosis, younger than 40 years, not fluent French speakers, not white, without a reliable informant, with a history of severe head trauma or neurosurgery, referred from another hospital, or not living in the urban community of Lille (1.2 million inhabitants). Previous stroke and TIA were not exclusion criteria. We examined patients according to a standardised procedure, as described previously. ${ }^{12}{ }^{17}$

\section{Patients' evaluation}

We prospectively collected demographic and clinical data, including personal history of epileptic seizures, age, sex, education level ( $<8$ years of education, 8 years of education), presence of arterial hypertension (defined as systolic blood pressure $>160 \mathrm{~mm} \mathrm{Hg}$ or diastolic blood pressure $>90 \mathrm{~mm}$ $\mathrm{Hg}$ or current treatment with antihypertensive drugs either before stroke onset or lasting more than one month after stroke onset), diabetes mellitus (defined as serum glucose concentration $>1.20 \mathrm{~g} /$ litre or current use of antidiabetic drugs), hyperlipidaemia (defined as fasting serum concentration of triglycerides $>1.5 \mathrm{~g}$ /litre or fasting cholesterol serum concentration $>2.3 \mathrm{~g} /$ litre), previous TIA or stroke, mean alcohol consumption $>300 \mathrm{~g} /$ week, and cigarette smoking (>10 cigarettes/day or cessation less than five years earlier). We evaluated the severity of the clinical deficits with the Orgogozo scale. $^{18}$ We determined the presence of silent infarcts on computerised tomography (CT) scans performed at admission without contrast agent, and the presence and severity of leucoaraiosis and cerebral atrophy with widely used criteria and scales previously described in this cohort. ${ }^{12}{ }^{17}$ The location of recent and old strokes was determined on the neuroimaging procedure judged as the most appropriate, which was either a delayed CT scan (87 patients) or a magnetic resonance imaging scan ( 82 patients). Thirty three patients had neither a delayed CT nor a magnetic resonance imaging scan because of death or refusal: in these patients, the location of stroke was determined on CT performed at admission. Lesions were considered to be superficial when they involved cortical areas and to be deep when they did not. In 24 patients, the stroke location was undetermined: 13 patients had no delayed imaging and stroke was not visible on the first CT scan and 11 patients had no visible lesion on delayed imaging. At discharge, the aetiology of ischaemic strokes was defined according to TOAST criteria. ${ }^{19}$

Abbreviations: $A D$, Alzeimer's disease; $\mathrm{Cl}$, confidence interval; $\mathrm{CT}$, computerised tomography; $\mathrm{ES}$, early seizures; IQCODE, Informant Questionnaire on Cognitive Decline in the Elderly; LS, late seizures; OR, odds ratio; TIA, transient ischaemic attack 


\section{Pre-existing dementia}

The assessment of pre-existing dementia was carried out within 48 hours of stroke onset by a French translation of the Informant Questionnaire on Cognitive Decline in the Elderly (IQCODE). ${ }^{20-24}$ We classified patients with IQCODE scores of 104 or more as having pre-existing dementia. ${ }^{20}$ This questionnaire consists of 26 questions regarding the changes experienced by the patient over the past 10 years in aspects of daily behaviour requiring memory and other intellectual abilities. A close relative is interviewed and therefore it does not require the participation of the patient at a stage of the disease when neuropsychological functions may be influenced by stroke. Each item carries a score of 1 to 5 ( 1 , has become much better; 2 , has become a bit better; 3 , has not changed; 4, has become a bit worse; and 5, has become much worse). The global score, achieved by adding the scores of each item, ranges from 26 to 130 points. The informant must have known the patient for at least 10 years and should meet him or her at least once a week. The questionnaire has a good reproducibility between and within raters. ${ }^{2022}{ }^{23}$ There is a good correlation between the scores of the Mini-Mental State Examination ${ }^{25}$ and the IQCODE. ${ }^{202324}{ }^{26}$ The IQCODE score is not influenced by patients' previous intelligence, education level, or socioeconomic class ${ }^{2022327} 28$ because its aim is to detect a fall from a higher previous to a lower present intellectual level. ${ }^{21}$ This screening tool has been validated not only in patients with Alzeimer's disease (AD), ${ }^{29}$ but also in population samples of elderly subjects. ${ }^{22}{ }^{24}{ }^{27}$ We classified as having pre-existing dementia those patients with IQCODE scores higher than 104, because this cutoff point leads to a diagnostic accuracy of $90.4 \%{ }^{20}$

\section{Seizures}

Seizures were classified according to the International League Against Epilepsy criteria. ${ }^{30}$ We only took into account those events that were certain to be seizures. We distinguished three groups: partial, partial secondary generalised, and generalised seizures. Early seizures (ES) were defined according to the guidelines of the International League Against Epilepsy ${ }^{31}$ as those occurring within seven days of stroke. Unprovoked seizures developing beyond one week of stroke were termed late seizures (LS). The use of antiepileptic treatment within seven days of stroke and at discharge was systematically recorded. LS, electroencephalogram data (if any), and current antiepileptic treatment were systematically recorded at each follow up visit.

\section{Follow up}

Patients were followed up at six months and then annually over three years by a visit to a neurologist. At each visit the occurrence of a recurrent stroke or TIA and of seizures was recorded.

\section{Ethics}

The promoter of the study was the Lille University Hospital, France. The study protocol was approved by the ethical committee of the institution. The study was considered by the ethical committee as observational, data recorded being part of the normal care and follow up of patients with stroke and no investigation being performed for the purpose of the study. The ethical committee disapproved the recruitment of a control group, which was initially planned, but therefore not recruited.

\section{Statistical analysis}

Clinical and imaging data of patients with and without ES after stroke were compared using the $\chi^{2}$ test, Fisher's exact test, or the $t$ test for unpaired data, as appropriate. We evaluated the influence of pre-existing dementia on the mortality rate within seven days of stroke onset using the $\chi^{2}$ test. Risk factors independently associated with ES were identified by means of logistic regression analysis. The incidence rate of LS was calculated using life table methods. Kaplan-Meier survival analysis was used to determine the proportion of patients surviving free of LS in those with and without pre-stroke dementia. The influence of demographic variables and of other potential predictors of LS was assessed using the log rank test for the following variables: demographic factors, vascular risk factors, previous stroke, type of stroke (ischaemic/haemorrhagic), aetiology of stroke according to TOAST criteria, ${ }^{19}$ topography of stroke (superficial, deep, posterior fossa), ES, and CT data (presence of silent infarcts on CT). The relative risk was computed using Cox proportional hazards analysis. Cox proportional hazards analysis was also used for qualitative variables (age, Orgogozo's score at admission, cerebral atrophy score, and leucoaraiosis score). Multivariate analysis was performed using Cox proportional hazards analysis including preexisting dementia, demographic variables (age and sex), and other potential predictors of LS. Only variables with $\mathrm{p}$ value $<0.20$ in the bivariate analysis were considered for multivariate analysis.

The analyses were computed using SPSS.

\section{RESULTS}

\section{Study population}

During the 28 week recruitment period, 258 patients with stroke, aged 40 years or more, French speakers, without a history of severe head trauma or neurosurgery were admitted into the acute stroke unit. Fifty six $(27.7 \%)$ were excluded because of the lack of an informant or the impossibility of meeting the informant within 48 hours of stroke onset. Those 56 subjects did not differ from the study population in age, sex, or severity of neurological deficit at admission. The study population consisted of 202 patients ( 105 women and 97 men), with a median age of 75 years (range, 42-101). Twenty five patients had a deep intracerebral haemorrhage and 177 an ischaemic stroke. One hundred and forty seven patients had a first ever stroke, and 32 had suffered from a previous stroke. No survivor was lost to follow up at month 36. Of the 202 patients, 33 (16.3\%; 95\% confidence interval (CI), 11.2 to 21.4 ) had an IQCODE score of 104 and were diagnosed as having pre-stroke dementia. Among 202 patients, only one patient had a seizure 30 years ago but did not have a seizure during the study.

\section{Early seizures}

Eleven (5.4\%) patients developed ES. Their median age was 76 years (range, 43-89). Three of these 11 patients had an intracranial haemorrhage and eight had an infarct, including one in the posterior fossa. In six patients, seizures occurred at onset, in two during the first 24 hours, in one at 48 hours, and in two patients seizures occurred five days after stroke onset. ES were classified as simple partial in five patients, secondary generalised in one, and generalised tonic-clonic without signs of focal onset in three. One patient suffered from an initial partial status epilepticus and the type of seizure could not be defined with certainty in one patient. Seizures were unique in all patients but one. Four patients received antiepileptic agents (carbamazepine in two, valproic acid in two). Table 1 details the results of the bivariate analyses regarding factors potentially associated with ES. The mortality rate within seven days after stroke was similar in patients with and without pre-existing dementia $(p=0.924)$. We found no independent predictor of ES in multivariate analysis. Pre-existing dementia was not associated with ES (odds ratio (OR), $1.11 ; 95 \% \mathrm{CI}, 0.26$ to 4.80 ), and neither was the IQCODE score when considered as a continuous variable $(\mathrm{p}=0.539)$. 
Table 1 Factors associated with early seizures: bivariate analysis

\begin{tabular}{|c|c|c|c|c|}
\hline Variable & $\begin{array}{l}\text { Patients without ES } \\
(n=191)\end{array}$ & $\begin{array}{l}\text { Patients with ES } \\
(n=11)\end{array}$ & $p$ Value & OR $(95 \% \mathrm{Cl})$ \\
\hline \multicolumn{5}{|l|}{ Demographic data } \\
\hline Age (years)* & $75(42-100)$ & $76(43-89)$ & 0.752 & \\
\hline Male sex & $91(47.6)$ & $6(54.5)$ & 0.761 & $1.14(0.43$ to 2.98$)$ \\
\hline \multicolumn{5}{|l|}{ Past medical history } \\
\hline Stroke & 30 (15.7) & $2(18.2)$ & 0.687 & $1.16(0.24$ to 5.51$)$ \\
\hline Alcohol abuse & 37 (19.4) & $3(27.3)$ & 0.458 & $1.38(0.40$ to 4.72$)$ \\
\hline \multicolumn{5}{|l|}{ Pre-existing cognitive status } \\
\hline Pre-existing dementia & $31(16.2)$ & $2(18.2)$ & 1.000 & $1.11(0.26$ to 4.80$)$ \\
\hline IQCODE score* & $83(78-130)$ & $85(78-121)$ & 0.539 & \\
\hline \multirow{2}{*}{\multicolumn{5}{|c|}{ Stroke severity }} \\
\hline Orgogozo's score at admission* & $70(0-100)$ & & & \\
\hline \multicolumn{5}{|l|}{ Type of stroke } \\
\hline \multirow{2}{*}{\multicolumn{5}{|c|}{$\begin{array}{l}\text { Ischaemic } \\
\text { Aetiology of ischaemic stroke }\end{array}$}} \\
\hline & & & & \\
\hline Atheroma & $26(13.6)$ & $1(9.1)$ & 1.000 & $0.68(0.09$ to 5.06$)$ \\
\hline Cardioembolic & $46(24.1)$ & $2(18.2)$ & 1.000 & $0.77(0.18$ to 3.34$)$ \\
\hline Small vessel disease & 35 (18.3) & $2(18.2)$ & 1.000 & $0.99(0.23$ to 4.30$)$ \\
\hline Other known cause & $4(2.1)$ & 0 & NA & NA \\
\hline Undetermined & $58(30.4)$ & $3(27.3)$ & 1.000 & $0.90(0.26$ to 3.13$)$ \\
\hline \multicolumn{5}{|l|}{ Location of the lesions (old and new) } \\
\hline Superficial & $78(40.9)$ & $3(27.3)$ & 0.531 & $0.68(0.19$ to 2.37$)$ \\
\hline Deep & $127(60.2)$ & $8(45.5)$ & 1.000 & $1.34(0.34$ to 5.24$)$ \\
\hline Posterior fossa & $18(9.4)$ & $1(9.1)$ & 1.000 & $0.97(0.13$ to 7.09$)$ \\
\hline \multicolumn{5}{|l|}{ Location of the lesions (old) } \\
\hline Superficial & $15(7.8)$ & $1(9.1)$ & 1.000 & 1. $16(0.14$ to 9.65$)$ \\
\hline Deep & $53(27.7)$ & $6(54.5)$ & 0.084 & $1.97(0.69$ to 5.58$)$ \\
\hline Posterior fossa & $10(5.2)$ & 0 & NA & NA \\
\hline \multicolumn{5}{|l|}{ Location of the lesions (new) } \\
\hline Superficial & $74(38.7)$ & $2(1.8)$ & 0.214 & $0.47(0.10$ to 2.18$)$ \\
\hline Deep & $115(60.2)$ & $5(45.5)$ & 0.359 & 0.75 (0.25 to 2.24 ) \\
\hline Posterior fossa & $18(9.4)$ & $1(9.1)$ & 1.000 & $0.96(0.12$ to 7.96$)$ \\
\hline \multicolumn{5}{|l|}{ Neuroimaging } \\
\hline Leucoaraiosis score & $1(0-3)$ & $2.5(0-3)$ & 0.071 & \\
\hline Cerebral atrophy score & $2(0-3)$ & $2(0-3)$ & 0.280 & \\
\hline
\end{tabular}

\section{Late seizures}

During 289 person-years of follow up, 14 patients developed LS, resulting in an incidence rate of 4.8 new cases/100 personyears. The median age was 66 years (range, 45-85). Two of the 14 patients suffered from an intracranial haemorrhage and 12 from an infarct. LS were classified as simple partial in four patients, secondary generalised in five, and generalised tonicclonic without signs of focal onset in five. The median delay between stroke and LS was 15.5 months (range, 4-63). None of the patients had a personal history of seizure. Only one patient had suffered from an ES. After the first episode of LS, eight patients were treated (valproic acid in four, carbamazepine in four). The remaining four received no treatment. This was an observational study and treatment decisions were made by the clinicians in charge of the patient. Before stroke onset, only one patient was known to have dementia, but was not treated. After stroke, this patient was treated with tacrine. He received the treatment over nine months and suffered from a first seizure seven months after stroke. He had a few recurrences of LS, which stopped when tacrine was withdrawn. No other patient received cholinesterase inhibitors during the follow up period.

LS occurred in four of 33 patients with pre-existing dementia and in 10 of the 169 without pre-existing dementia.

Tables 2 and 3 detail the results of the bivariate analyses for qualitative and quantitative variables, respectively. Multivariate analysis found patients with pre-existing dementia to have a higher risk of LS (adjusted OR, 4.66; $95 \%$ CI, 1.34 to 16.21 ). The only other independent risk factor for LS was the clinical severity of the stroke at admission (adjusted OR, 0.98; 95\% CI, 0.95 to 0.99 ; goodness of fit, 89.49).

\section{DISCUSSION}

Our study has shown that patients with pre-existing dementia have an increased risk of post-stroke LS, but not of ES.

To our knowledge, this is the first study to investigate the influence of pre-existing dementia on the risk of post-stroke epileptic seizures. Pre-existing dementia does not increase the risk of ES but increases the risk of LS. The absence of an association between pre-existing dementia and ES cannot be explained by a higher mortality rate in patients with preexisting dementia because the mortality rate within seven days after stroke onset was similar in patients with and without pre-existing dementia. With regard to the influence of pre-existing dementia on the risk of LS, because of the high mortality rate in this population (in particular patients with pre-existing dementia) ${ }^{12}$ we used survival curve analyses to determine the predictive factors for LS. In a population based study, ${ }^{32}$ patients with dementia (mainly suffering from $\mathrm{AD}$ ) were found to have an increased risk of seizures compared with those without dementia. Studies dedicated to the population of patients with dementia ${ }^{15}{ }^{16}$ suggested that seizures occur at the late stage of dementia. To date, no clear pathophysiological pattern is available to explain the occurrence of seizures in the course of dementia. Dysfunction of excitatory amino acid pathways, such as glutamate, may play a role. ${ }^{33}$ In our population, patients with dementia were mainly diagnosed as having $\mathrm{AD}^{,{ }^{12}}$ which can, to some extent, explain the relation between pre-existing dementia and seizures.

We used the IQCODE to diagnose pre-existing dementia. To prevent an overestimation of the prevalence of dementia, we chose a cutoff point of 104 for the IQCODE score, as Jorm 
Table 2 Factors associated with LS: bivariate analysis (Kaplan-Meier analysis, log rank test)

\begin{tabular}{|c|c|c|c|c|}
\hline Variable & $\begin{array}{l}\text { No of patients } \\
(n=202)\end{array}$ & $\begin{array}{l}\text { No of patients with LS } \\
(n=14)\end{array}$ & p Value & $\operatorname{RR}(95 \% \mathrm{Cl})$ \\
\hline \multicolumn{5}{|l|}{ Demographic data } \\
\hline Male & 97 & 5 & 0.077 & $0.32(0.09$ to 1.21$)$ \\
\hline Female & 105 & 9 & & \\
\hline \multicolumn{5}{|l|}{ Past medical history } \\
\hline Previous stroke & 32 & 4 & 0.378 & $1.76(0.49$ to 6.29$)$ \\
\hline No previous stroke & 170 & 10 & 0.156 & $0.25(0.03$ to 1.25$)$ \\
\hline Alcohol abuse & 40 & 2 & & \\
\hline No alcohol abuse & 162 & 12 & & \\
\hline \multicolumn{5}{|l|}{ Pre-existing cognitive status } \\
\hline Pre-existing dementia & 33 & 4 & 0.016 & 4.14 (1.20 to 14.29$)$ \\
\hline No pre-existing dementia & 169 & 10 & & \\
\hline \multicolumn{5}{|l|}{ Type of stroke } \\
\hline Ischaemic & 177 & 12 & 0.719 & 0.75 (0.16 to 3.54$)$ \\
\hline Haemorrhagic & 25 & 2 & & \\
\hline \multicolumn{5}{|l|}{ Aetiology of ischaemic stroke } \\
\hline Atheroma & 27 & 1 & 0.321 & 0.36 (0.04 to 2.92 ) \\
\hline No atheroma & 175 & 13 & & \\
\hline Cardioembolic & 48 & 1 & 0.513 & 0.51 (0.07 to 3.99$)$ \\
\hline Not cardioembolic & 154 & 13 & & \\
\hline Small vessel disease & 37 & 2 & 0.090 & 0.21 (0.03 to 1.62$)$ \\
\hline No small vessel disease & 165 & 12 & & \\
\hline Other known cause & 4 & 0 & NA & NA \\
\hline No other known cause & 198 & 14 & & \\
\hline Undetermined & 61 & 8 & 0.004 & 5.72 (1.52 to 21.56$)$ \\
\hline Not undetermined & 141 & 6 & & \\
\hline \multicolumn{5}{|l|}{ Location of the lesions (old) } \\
\hline Superficial & 16 & 2 & 0.010 & 5.97 (1.26 to 28.32$)$ \\
\hline Not superficial & 186 & 12 & & \\
\hline Deep & 59 & 5 & 0.897 & $1.08(0.34$ to 3.37$)$ \\
\hline Not deep & 143 & 9 & & \\
\hline Posterior fossa & 10 & 1 & 0.351 & $2.57(0.33$ to 20.21$)$ \\
\hline Not in the posterior fossa & 192 & 13 & & \\
\hline \multicolumn{5}{|l|}{ Location of the lesions (new) } \\
\hline Superficial & 76 & 7 & 0.008 & 4.52 (1.32 to 15.44$)$ \\
\hline Not superficial & 126 & 7 & & \\
\hline Deep & 120 & 8 & 0.487 & $0.68(0.23$ to 2.04$)$ \\
\hline Not deep & 82 & 6 & & \\
\hline Posterior fossa & 19 & 1 & 0.733 & 0.70 (0.09 to 5.48$)$ \\
\hline Not in the posterior fossa & 183 & 13 & & \\
\hline \multicolumn{5}{|c|}{ Location of the lesions (old and new) } \\
\hline Superficial & 81 & 7 & 0.017 & 4.01 (1.17 to 13.75$)$ \\
\hline Not superficial & 121 & 7 & & \\
\hline Deep & 135 & 11 & 0.927 & $1.06(0.28$ to 4.02$)$ \\
\hline Not deep & 67 & 3 & & \\
\hline Posterior fossa & 28 & 2 & 0.757 & $0.72(0.09$ to 5.65$)$ \\
\hline Not in the posterior fossa & 174 & 12 & & \\
\hline \multicolumn{5}{|l|}{ Occurrence of seizures } \\
\hline Early & 11 & 1 & 0.793 & 0.74 (0.08 to 7.23$)$ \\
\hline Not early & 191 & 13 & & \\
\hline
\end{tabular}

and Jacomb did in their first work. ${ }^{20}$ For the French version, the optimal cutoff point to diagnose dementia is probably lower. Therefore, we may have underestimated the prevalence of pre-existing dementia. However, this method is as sensitive as the Mini-Mental State Examination ${ }^{25}$ for the diagnosis of moderate dementia.

Pre-existing dementia, as defined by an IQCODE score higher than 104, was associated with LS. When analysing the IQCODE score as a continuous variable, the association with
LS did not reach significance in bivariate analysis. This suggests that a certain degree of severity of cognitive decline is needed to influence the risk of epileptic seizures.

The incidence of ES was $5.4 \%$ and 4.8 new cases/100 person-years for LS, which is similar to the figures reported in previous studies. ${ }^{8}$ This finding suggests that the study population and the method used to define seizures did not differ from other studies, and therefore, did not influence the result of the analysis.

Table 3 Factors associated with late seizures: bivariate analysis for quantitative variables (Cox proportional hazard model)

\begin{tabular}{lllll}
\hline Variable & $\boldsymbol{\beta}$ & SE & p Value & HR (95\% Cl) \\
\hline Age & -0.010 & 0.023 & 0.642 & $0.99(0.95$ to 1.03$)$ \\
Orgogozo's score at admission & -0.028 & 0.011 & 0.009 & $\mathbf{0 . 9 7}(0.95$ to 0.99$)$ \\
Leucoaraiosis score & 0.087 & 0.281 & 0.757 & $1.09(0.63$ to 1.89$)$ \\
IQCODE score & 0.030 & 0.020 & 0.130 & $1.03(0.99$ to 1.07$)$ \\
Cerebral atrophy score & 0.009 & 0.314 & 0.976 & $1.01(0.55$ to 1.87$)$ \\
\hline
\end{tabular}

Results in bold are significant.

$\mathrm{Cl}$, confidence interval; $\mathrm{HR}$, hazards ratio; IQCODE, Informant Questionnaire on Cognitive Decline in the Elderly. 
The assessment of seizures was not blinded for the presence or absence of pre-existing dementia because the assessment of both dementia and seizures was performed by the same neurologist. Although we cannot completely exclude an influence on the results, this is very unlikely.

Some of the patients studied had a medical history of stroke. We chose to include them because recurrence of stroke is frequent and an important issue in practice; we took this into account in our statistical analysis. A medical history of stroke was not significant. We also studied the influence of existing lesions on imaging: in multivariate analysis, neither old nor new lesions were a predictive factor for post-stroke seizures.

Our study was conducted between 1995 and 1999. In France, donepezil was released in 1997 and rivastigmin in 1998. At the beginning, cholinesterase inhibitors were used in patients who fulfilled the criteria for probable $\mathrm{AD}$, but not in patients with possible AD. Because all our patients had suffered from a stroke, even if the clinical presentation and course of dementia was typical of $\mathrm{AD}$, they received the diagnosis of possible $\mathrm{AD}$ and not of probable $\mathrm{AD}$. This may explain why only one patient was treated with cholinesterase inhibitors in our cohort.

After the first LS, one third of patients did not receive antiepileptic drugs. Our study was observational, and not designed to determine the reasons for prescription (or nonprescription). Treatment decisions were made by the clinicians in charge of the patients, so we cannot determine why some patients with LS did not receive anti-epileptic treatment. However, to date, there is no consensus regarding the indications for anti-epileptic drugs after a first post-stroke late seizure.

We found no explanatory variables regarding the occurrence of ES. One of the reasons may result from the time definition of ES. According to the international league of epilepsy, seizures occurring within seven days are considered to be early. ${ }^{31}$ The pathophysiological mechanisms of seizures that occur at onset may differ from those occurring five days after the stroke. Gathering those different types of seizures in the same analysis might explain the difficulty of identifying strong predictors for ES. We did not analyse separately those seizures occurring at stroke onset and those occurring after 24 hours because of the small population size. Other factors such as hyperthermia, electrolyte disturbances, and treatments may play a role in facilitating seizures; we did not take them into account because of the small size of our study population.

The difficulty in identifying predictive factors for seizures or the characteristics of stroke responsible for seizures may result from the fact that seizures are not only linked to the stroke in itself, but are also the consequence of host characteristics. From a practical point of view, besides giving information to patients and caregivers about the risk of stroke recurrence, post-stroke dementia, or depression, we should also inform patients and caregivers about the risk of post-stroke late seizures, especially in patients with preexisting dementia. Any factor increasing the risk of seizures (drugs, metabolic changes) should be avoided in these patients.

\footnotetext{
Authors' affiliations

C Cordonnier, H Hénon, D Leys, Department of Neurology, Stroke Unit, University of Lille, Lille F-59037, France

F Pasquier, Memory Clinic, University of Lille

P Derambure, Clinical Neurophysiology Department, University of Lille

Conflicting interests: none declared
}

Although Professor D Leys is an associate editor of the journal, the manuscript was handled by M Rossor, editor in chief. D Leys did not participate in the editorial process for this manuscript.

\section{REFERENCES}

1 Kilpatrick C, Davis SM, Tress BM, et al. Epileptic seizures in acute stroke. Arch Neurol 1990;47:157-60.

2 Giroud M, Gras P, Fayolle H, et al. Early seizures after acute stroke: a study of 1640 cases. Epilepsia 1994;35:959-64.

3 Lancman M, Golimstok A, Norscini J, et al. Risk factors for developing seizures after a stroke. Epilepsia 1993;34:141-3.

4 Reith J, Jorgensen HS, Nakayama H, et al. Seizures in acute stroke: predictors and prognostic significance. The Copenhagen stroke study. Stroke 1997:28:1585-9.

5 Berges S, Moulin T, Berger E, et al. Seizures and epilepsy following strokes: recurrences factors. Eur Neurol 2000;43:3-8.

6 Bladin C, Alexandrov AV, Bellavance A, et al. Seizures after stroke: a prospective multicenter study. Arch Neurol 2000;57:1617-22.

7 Lamy C, Domigo V, Semah F, et al. Early and late seizures after cryptogenic ischemic stroke in young adults. Neurology 2003;60:400-4

8 Camilo O, Goldstein LB. Seizures and epilepsy after ischemic stroke. Stroke 2004;35: 1769-75.

9 Burn J, Dennis M, Bamford J, et al. Epileptic seizures after a first stroke: the Oxfordshire community stroke project. BMJ 1997;315:1582-7.

10 So E, Annegers JF, Hauser WA, et al. Population-based study of seizure disorders after cerebral infarction. Neurology 1996:46:350-5.

11 Lo Y, Yiu CH, Hu HH, et al. Frequency and characteristics of early seizures in Chinese acute stroke. Acta Neurol Scand 1994;90:83-5.

12 Hénon $\mathrm{H}$, Pasquier $\mathrm{F}$, Durieu $\mathrm{l}$, et al. Preexisting dementia in stroke patients: baseline frequency, associated factors, and outcome. Stroke 1997;28:2429-36

13 Barba R, Castro MD, del Mar Morin M, et al. Prestroke dementia. Cerebrovasc Dis 2001;11:216-24.

14 Klimkowicz A, Dziedzic T, Slowik A, et al. Incidence of pre- and poststroke dementia: Cracow stroke registry. Dement Geriatr Cogn Disord 2002; 14:137-40.

15 Volicer L, Smith S, Volicer BJ. Effect of seizures on progression of dementia of the Alzheimer type. Dementia 1995;6:258-63.

16 Hesdorffer D, Hauser WA, Annegers JF, et al. Dementia and adult-onset unprovoked seizures. Neurology 1996;46:727-30.

17 Hénon H, Durieu I, Guerouaou D, et al. Poststroke dementia. Incidence and relationship to prestroke cognitive decline. Neurology 2001;57:1216-22.

18 Orgogozo J, Dartigues JF. Methodology of clinical trials in acute trials in acute cerebral ischemia: survival, functional and neurological outcome measures. Cerebrovasc Dis 1991;1:100-11.

19 Adams HJ, Bendixen BH, Kappelle $\amalg$, et al. Classification of subtypes of acute ischemic stroke: definitions for use in a multicenter clinical trial. Stroke 1993;24:35-41.

20 Jorm A, Jacomb PA. The informant questionnaire on cognitive decline in the elderly (IQCODE): socio-demographic correlates, reliability, validity and some norms. Psychol Med 1989;19:1015-22.

21 Jorm A, Korten AE. Assessment of cognitive decline in the elderly by informant interview. Br J Psychiatry 1988;152:209-13.

22 Jorm A, Broe GA, Creasey $H$, et al. Further data on the validity of the Informant Questionnaire on Cognitive Decline in the Elderly (IQCODE). Int J Geriatric Psychiatry 1996;1 1:131-9.

23 Jorm A, Scott R, Cullen JS, et al. Performance of the informant questionnaire on cognitive decline in the elderly (IQCODE) as a screening test for dementia. Psychol Med 1991;21:785-90.

24 Mulligan R, Mackinnon A, Jorm AF, et al. A comparison of alternative methods of screening for dementia in clinical settings. Arch Neurol 1996;23:532-6.

25 Folstein M, Folstein SE, McHugh PR. "Mini Mental State": a practical method for grading the cognitive state of patients for the clinician. J Psychiatr Res 1975; 12:189-98

26 Bowers J, Jorm AF, Henderson S, et al. General practitioner's detection of depression and dementia in elderly patients. Med J Aust 1990;153:192-6.

27 Morales J, Gonzalez-Montalvo Jl, Bermejo F, et al. The screening of mild dementia with a shortened version on the Informant Questionnaire on Cognitive Decline in the Elderly. Alzheimer Dis Assoc Disord 1995;9:105-11.

28 Withdrawn.

29 Law S, Wolfson C. Validation of a French version of an informant-based questionnaire as a screening test for Alzheimer's disease. Br J Psychiatry 1995; $167: 541-4$

30 Commission of Classification and Terminology International League Against Epilepsy. Proposal for revised clinical and electroencephalographic classification of epileptic seizures. Epilepsia 1981;22:489-501.

31 Commission on Epidemiology and Prognosis International League Against Epilepsy. Guidelines for epidemiologic studies on epilepsy. Epilepsia 1993;34:592-6.

32 Hauser W, Annegers JF, Kurland LT. Incidence and clinical characterization of unprovoked seizures in Rochester, Minnesota; 1935-1984. Epilepsia 1993;34:453-68.

33 Greenamyre J, Young AB. Excitatory amino acids and Alzheimer's disease. Neurobiol Aging 1989; 10:593-602. 